\title{
Assessing the impact of exact reads on reducing the error rate of read mapping
}

\author{
Farzaneh Salari ${ }^{1}$, Fatemeh Zare-Mirakabad ${ }^{1}{ }^{2 *}$, Mehdi Sadeghi ${ }^{3}$ and Hassan Rokni-Zadeh ${ }^{4}$
}

\begin{abstract}
Background: Nowadays, according to valuable resources of high-quality genome sequences, reference-based assembly methods with high accuracy and efficiency are strongly required. Many different algorithms have been designed for mapping reads onto a genome sequence which try to enhance the accuracy of reconstructed genomes. In this problem, one of the challenges occurs when some reads are aligned to multiple locations due to repetitive regions in the genomes.

Results: In this paper, our goal is to decrease the error rate of rebuilt genomes by resolving multi-mapping reads. To achieve this purpose, we reduce the search space for the reads which can be aligned against the genome with mismatches, insertions or deletions to decrease the probability of incorrect read mapping. We propose a pipeline divided to three steps: ExactMapping, InExactMapping, and MergingContigs, where exact and inexact reads are aligned in two separate phases. We test our pipeline on some simulated and real data sets by applying some read mappers. The results show that the two-step mapping of reads onto the contigs generated by a mapper such as Bowtie2, BWA and Yara is effective in improving the contigs in terms of error rate.

Conclusions: Assessment results of our pipeline suggest that reducing the error rate of read mapping, not only can improve the genomes reconstructed by reference-based assembly in a reasonable running time, but can also have an impact on improving the genomes generated by de novo assembly. In fact, our pipeline produces genomes comparable to those of a multi-mapping reads resolution tool, namely MMR by decreasing the number of multi-mapping reads. Consequently, we introduce EIM as a post-processing step to genomes reconstructed by mappers.
\end{abstract}

Keywords: Reference-based assembly, Read mapping, Multi-mapping reads

\section{Background}

The advent of next generation sequencing (NGS) technologies by greatly increasing the volume of produced data, created a genomic revolution. Massive amount of data and low cost of these technologies make it possible to determine large parts of a genome sequence in a short time. Today, biological research on any organism from viruses and bacteria to humans depends on the genome sequence information. In addition, sequences of organisms have an important role in understanding diseases.

*Correspondence: f.zare@aut.ac.ir

${ }^{1}$ Mathematics and Computer Science Department, Amirkabir University of Technology (Tehran polytechnic), Tehran, Iran

${ }^{2}$ School of Biological Science, Institute for Research in Fundamental Sciences (IPM) P.O. Box: 19395-5746, Tehran, Iran

Full list of author information is available at the end of the article
In order to reconstruct a genome sequence based on NGS data, genome assembly, one of the challenging problems in bioinformatics, is defined. There are two different approaches to model genome assembly: de novo and reference-based assembly. In the first model, a novel genome sequence is reconstructed from scratch by only applying NGS reads. In the second one, a reference genome is employed to assemble the NGS reads by mapping them onto the reference.

Because of the large volume of NGS reads, established alignment algorithms such as Smith-Waterman aren't efficient for read mapping. To reduce search space, several algorithms have been developed [1-5] using the seedand-extending approach in which the reads are mapped onto the reference in two main steps. Firstly, some subsequences of each read are selected as seeds to find their 
positions in the reference. In this way, the candidate locations of the reads are determined rapidly. Secondly, each read is aligned to its candidate locations by a dynamic programming algorithm in order that the actual mapping positions are obtained.

During the past years, various algorithms have been designed to improve the accuracy and efficiency of mappers [6-13]. Although these algorithms represent appropriate approaches to reduce the time and space complexity, resolving multi-mapping reads in genome reconstruction has remained a challenge. Due to repetitive regions within the genome, some reads can be mapped to multiple locations of the reference genome. Multi-mapping reads may be aligned at incorrect locations since the read set contains sequencing errors and genetic variations relative to the reference. As a result, some errors such as mismatches and indels (insertions or deletions) are introduced to the reconstructed genome. Read mappers often randomly select one of the locations for a multi-mapping read as the primary one. Recently, a post-processing tool (MMR) has been developed [14] to find optimal locations for multi-mapping reads within DNA- and RNA-seq alignment results. It resolves the problem based on the assumption of aligned reads coverage uniformity.

In this study, we introduce a new view to resolving multi-mapping reads by increasing the rate of reads aligned uniquely to the reference in order to decrease the error rate of the reconstructed genome sequence. For this aim, we divide the reads into two groups in accordance with the reference genome. The idea is inspired by the following fact.

Consider a target genome (the genome from which a set of reads is sampled) which is highly similar to the respective reference genome. If the read set is mapped onto the reference, high percentage of the reference can be covered by the reads uniquely aligned without mismatches and indels (exact reads). Leftover alignable reads (inexact reads) are then mapped to the remaining parts of the reference. Therefore, to reconstruct most of the target genome, it is enough to find the locations of reads which have unique exact-matching with the reference. The rest of the target genome can be rebuilt by aligning remaining reads against the reference with mismatches and indels.

Most of the existing read mappers don't consider any differences between the mapping of exact and inexact reads. For example, hash-based mappers find seeds which support mismatches (space-seeds) and gaps on the whole reference genome for all reads [15]. On the one hand, consecutive seeds are enough for exact reads and using space-seeds leads to excessive memory consumption. On the other hand, inexact reads are aligned by finding candidate locations on the whole reference genome, while according to high similarity between a target genome and its reference, searching in small parts of the reference is sufficient to find these types of reads.

Based on defining reads in two types: exact and inexact reads, we present a pipeline (EIM - mapping Exact and Inexact reads separately and then Merging the constructed contigs) for resequencing of a genome. To assess our pipeline, we have chosen Bowtie2 [7] as a highly cited and user-friendly mapper and used some real and simulated read sets. For a more complete evaluation of EIM pipeline, two other mappers are also used. Our results illustrate that EIM pipeline improves the quality of genomes reconstructed by the mappers in terms of error rate and yields comparable results to MMR in reducing errors.

\section{Methods}

Let $S=s_{1} s_{2} \ldots s_{L}$ denote a DNA sequence in which $\forall_{1 \leq i \leq L} \quad s_{i} \in\{A, C, G, T, N\}$; and $|S|$ denote the length of $S$. A genome sequence is a long DNA sequence. A set of paired reads is defined as $R=$ $\left\{\left\langle r_{1}, r_{1}^{\prime}\right\rangle,\left\langle r_{2}, r_{2}^{\prime}\right\rangle, \ldots,\left\langle r_{m}, r_{m}^{\prime}\right\rangle\right\}$ where for each $i, r_{i}$ and $r_{i}^{\prime}$ are short DNA sequences with length of $k$.

We propose a three-step pipeline (Fig. 1) for referencebased assembly as below, where a set of paired reads $R$ and a genome sequence $G$ are given as inputs:

\section{i. ExactMapping}

The set of reads is mapped onto the genome sequence without mismatches and indels. Then an exact contig set called $C n g 1$ is generated from uniquely mapped reads.

\section{ii. InExactMapping}

The remaining reads from previous step are mapped onto the regions of the genome which are covered with no contigs of $C n g 1$ to construct an inexact contig set named Cng2.

\section{iii. MergingContigs}

The two contig sets, Cng1 and Cng2 are merged to build up ultimate contigs.

In the following, each step of EIM pipeline is described in detail.

\section{ExactMapping}

In this step, we should apply a mapper to align the set of reads with the genome without mismatches and indels. In this regard, the genome $G$ and the read set $R$ are given to the mapper as inputs. After running the mapper, two outputs are produced: $i$ ) set $R^{\prime} \subset R$ containing unmapped and multi-mapping reads $i i$ ) SAM file [16] including the information of the alignment. Then consensus sequence $C$ is built up from uniquely mapped reads in the SAM file, where $C$ is a DNA sequence with length $|G|$. Afterwards, a set of contigs called $C n g 1$ is generated by breaking the sequence $C$ at each position of ' $N$ '. 


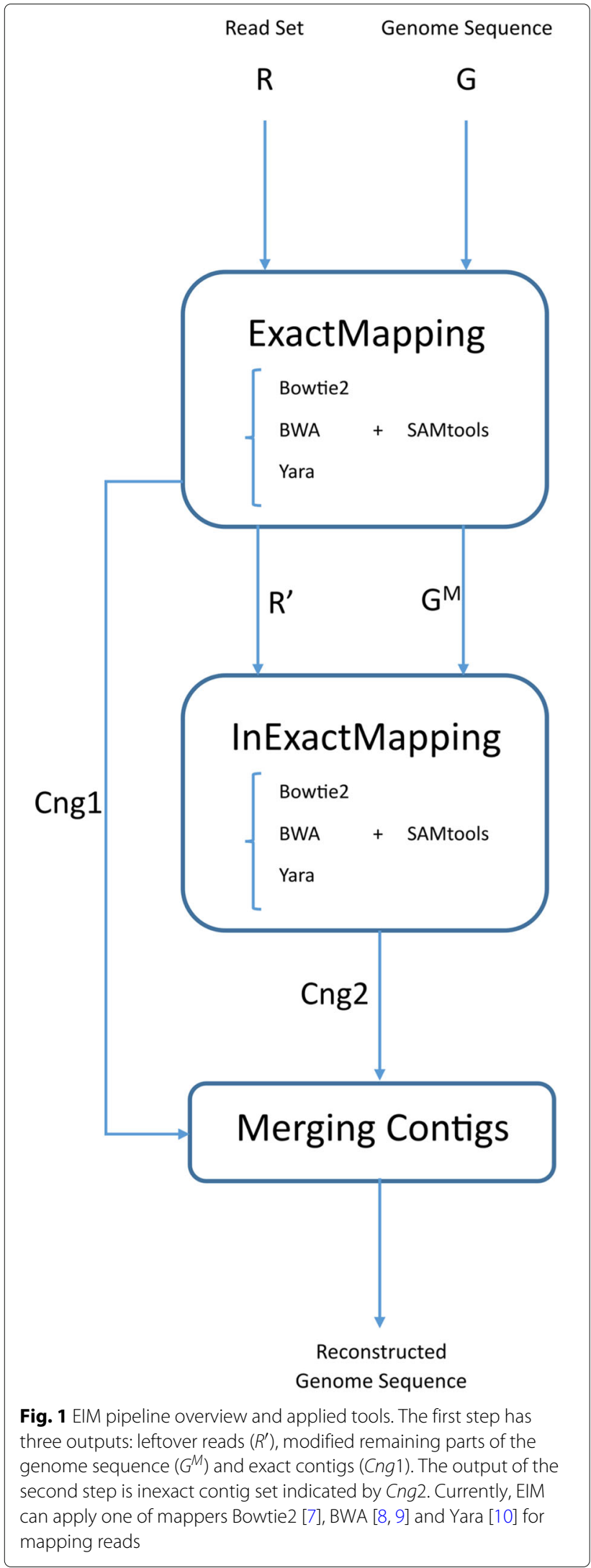

\section{InExactMapping}

In this stage, genome sequence $G=g_{1} g_{2} \ldots g_{n}$ is modified based on consensus sequence $C=c_{1} c_{2} \ldots c_{n}$ to generate a new genome called $G^{M}$. To construct genome $G^{M}$, the following steps are taken:

1: Make sequence $C^{\prime}=c_{1}^{\prime} c_{2}^{\prime} \ldots c_{n}^{\prime}$ as follows:

$$
c_{i}^{\prime}= \begin{cases}N & c_{i} \in\{A, C, G, T\}, \\ g_{i} & c_{i}=N,\end{cases}
$$

where $C^{\prime}$ contains all parts of genome $G$ covered with no contigs of Cng1.

2: Generate sequence $G^{M}=g_{1}^{M} g_{2}^{M} \ldots g_{n}^{M}$ by extending each contiguous nucleic acid sequence as:

$$
g_{i}^{M}=\left\{\begin{array}{ll}
c_{i}^{\prime} & c_{i}^{\prime} \in\{A, C, G, T\}, \\
g_{i} & c_{i}^{\prime}=N \& \exists_{j=1}^{k} c_{i \pm j}^{\prime} \\
N & \text { o.w }
\end{array} \in\{A, C, G, T\},\right.
$$

where $k$ is equal to the read length.

Then $G^{M}$ is broken at each position of 'N', and as a result a set of contigs is obtained. After that, a mapper is used in order to align $R^{\prime}$ against the set of contigs with mismatches and indels. Finally, a consensus sequence is made from mapped reads in the SAM file for each contig and added to Cng2.

\section{MergingContigs}

In this part, the two contig sets $C n g 1$ and $C n g 2$ generated respectively at the steps of ExactMapping and InExactMapping, are combined to rebuild the target genome. Although Cng1 contains large contigs which make up most of the target genome, $C n g 2$ is required to produce larger contigs including the differences with genome $G$. We merge the contig sets without alignment because the positions of contigs relative to the genome $\mathrm{G}$ are known. In this way, every two contigs of Cng1 are joined by a contig of $\mathrm{Cng} 2$ overlapping with both of them. Merging method is described in more detail below.

The union of Cng1 and Cng2 contig sets is defined as Cng $=\left\{\prec D^{i}, s^{i}, e^{i}, t^{i} \succ \mid D^{i}=d_{1}^{i} d_{2}^{i} \cdots d_{e^{i}-s^{i}+1}^{i}\right\}$ where for each $i, D^{i}$ is a contig belonging to either Cng1 or Cng2. The start and end positions of contig $D^{i}$ on the reference are shown by $s^{i}$ and $e^{i}$, repectively. It should be noted that $s^{i}<s^{i+1}$ and $e^{i}<e^{i+1}$. Moreover, the value of $t^{i}$ is set to 1 (or 2) when $D^{i} \in C n g 1$ (or $D^{i} \in C n g 2$ ). In the following, all the contigs in $\mathrm{Cng}$ are merged by a recursive equation: 


$$
m^{i}=\left\{\begin{array}{lll}
\emptyset & i=0, \\
m^{i-1} \cdot D^{i} & 1 \leq i \leq|\operatorname{Cng}| \& t^{i}=1, & \text { (1a) } \\
m^{i-1} \cdot d_{1}^{i} d_{2}^{i} \cdots d_{\left(e^{i}-s^{i}+1\right)-k}^{i} & i=1 \& t^{i}=2, & \text { (1b) } \\
m^{i-1} \cdot d_{k+1}^{i} d_{k+2}^{i} \cdots d_{\left(e^{i}-s^{i}+1\right)-k}^{i} & 1<i<|\operatorname{Cng}| \& t^{i}=2, & \text { (1c) } \\
m^{i-1} \cdot d_{k+1}^{i} d_{k+2}^{i} \cdots d_{\left(e^{i}-s^{i}+1\right)}^{i} & i=|C n g| \& t^{i}=2, & \text { (1d) }
\end{array}\right.
$$

where $k$ is equal to the length of a read, and $|C n g|$ is the number of contigs in the Cng set. For each $\mathrm{i}, \mathrm{m}^{i}$ denotes the merged sequence achieved by combining $D^{1}$ to $D^{i}$. Part (1a) of the above equation shows that each contig of Cng 1 has to be completely inserted to the merged sequence as it is highly probable that the contig has been made correctly. Parts (1b), (1c) and (1d) indicate how to insert a contig of $\mathrm{Cng} 2$ to the merged sequence after removing the extended parts (with length of $k$ ). The ultimate merged sequence is represented by $m^{|C n g|}$ which may include some Ns because of $C n g 2$ contigs. Thus $m^{|C n g|}$ sequence is broken at each position of ' $\mathrm{N}$ ' for generating output contigs of EIM pipeline.

\section{Datasets}

Several real and simulated datasets are used to evaluate the accuracy of EIM pipeline. The first real dataset is an Illumina MiSeq pair-end read set from $E$. coli downloaded from $[17,18]$ which consists of about 1.5 million paired reads of 151 base-pair (bp) with coverage depth $100 \times$. We apply Escherichia coli str. K12 substr. MG1655 [GenBank:NC_000913] as a reference genome and Escherichia coli O145:H28 str. RM12581 [GenBank: CP007136.1] as a related strain.

The second dataset includes four human chromosome read sets: Chr1, Chr10, Chr14 and Chr21 extracted from samples. The whole human genome samples are downloaded from the SRA database of National Center for Biotechnology Information (NCBI) with accession numbers SRR67780, SRR67785, SRR67787, SRR67789, SRR67791, SRR67792, SRR67793. The human reference genome GRCh38 is downloaded from [19]. All read sets contain 101 bp paired reads with the properties shown in Table 1.

We simulate several read sets for a prokaryotic and eukaryotic genome: E. coli and Arabidopsis thaliana. To simulate reads for $E$. coli, we create four genome sequences, E. coli-Mut1 to E. coli-Mut4 derived from $E$. coli K12. Then Illumina read sets, ReadSet1 to ReadSet8 and ReadSet 9 to ReadSet 12 are simulated for mutated

Table 1 Real data sets properties

\begin{tabular}{llll}
\hline Data set & GenomeLength & Reads\# & Coverage \\
\hline Human Chr 1 & $248,956,422$ & $80,623,200$ & $34 \times$ \\
Human Chr 10 & $133,797,422$ & $45,121,800$ & $34 \times$ \\
Human Chr 14 & $107,349,540$ & $36,117,398$ & $42 \times$ \\
Human Chr 21 & $46,709,983$ & $18,941,800$ & $42 \times$ \\
\hline
\end{tabular}

genomes by DWGSIM [20] and ART [21], respectively. E. coli-Mut1 and E. coli-Mut2 have single nucleotide variants (SNVs) with the rate of $0.1 \%$. E. coli-Mut2 has SNVs of random size among 1 to 3. E. coli-Mut3 has SNVs and deletions of the rates $0.09 \%$ and $0.01 \%$ respectively. E. coliMut4 has SNVs and insertions of the rates $0.09 \%$ and $0.01 \%$ respectively. The read sets, ReadSet 1 to ReadSet 4 are simulated such that the length and coverage depth of the reads are similar to those of the real read set from $E$. coli $K 12$ genome (i. e. more than 1.5 million paired reads of $150 \mathrm{bp}$ ). The read sets, ReadSet 5 to ReadSet12 are simulated with low coverage (i. e. about 3000 paired reads of $150 \mathrm{bp}$ ) and sequencing error. The properties of simulated reads are shown in Table 2 .

To generate reads for Arabidopsis thaliana, we create a genome sequence derived from TAIR10 [GenBank: CP002684.1-CP002688.1] reference genome. Firstly, TAIR10 genome sequence is mutated based on bur- 0 strain variations obtaining from [22]. Then an Illumina read set including 15.6 million paired reads of 150 bp with coverage depth of $20 \times$ is simulated by ART simulator.

\section{Tools}

Some tools are utilized for running EIM pipeline as follows. We use DWGSIM [20] and ART [21] for simulating reads, Bowtie2, Yara [10] and BWA $[8,9]$ for mapping reads, and SAMtools [16] for making consensus sequences. We also implement a simple hash-based aligner called ExactMapper for mapping reads without mismatches and gaps to make the pipeline faster.

The assessments on large genomes including human chromosomes and Arabidopsis thaliana are performed on a desktop which has a $3.60 \mathrm{GHz}$ Intel(R) Core(TM) $i 7-6850 \mathrm{~K}$ 6-core processor and 32GB of RAM running 64-bit Ubuntu 18.04 LTS. The other assessments are performed on a laptop with an Intel(R) Core(TM) $i 7-3517 \mathrm{U}$ processor and 8GB of RAM running 64-bit Ubuntu 15.10.

At ExactMapping step, we apply ExactMapper aligner for small genomes to generate a SAM file and extract remaining reads (unmapped and multi-mapping reads) simultaneously. Next, SAM file is given to a script to build up a consensus sequence $C$ from uniquely mapped reads. At InExactMapping step, we employ one of the aforementioned mappers with appropriate parameters and then construct the consensus sequence by SAMtools. For this purpose, The '--keep-masked-ref' parameter is set for 'bcftools call' command of SAMtools to be able to make consensus in IUPAC positions of the reference genome.

It should be noted, for large genomes such as the human chromosome 14, we use Bowtie2 in ExactMapping step. The '--score-min' parameter of Bowtie2 is set to the value ' $C, 0,-1$ ' to only map the reads with exact matches to the genome. Th unmapped and multi-mapping reads are 
Table 2 Simulated data sets properties

\begin{tabular}{llllllllll}
\hline Data set & Target genome & Genome length & Indel+SNV \% & \# SNVs & \# Insertions & \# Deletions & Read length & Coverage & Simulator \\
\hline ReadSet1 & E. coli-Mut1 & 4639675 & 0.1 & 4640 & 0 & 0 & 150 & $100 \times$ & DWGSIM \\
ReadSet2 & E. coli-Mut2 & 4639675 & 0.1 & 4640 & 0 & 0 & 150 & $100 \times$ & DWGSIM \\
ReadSet3 & E. coli-Mut3 & 4639138 & 0.1 & 4205 & 0 & 537 & 150 & $100 \times$ & DWGSIM \\
ReadSet4 & E. coli-Mut4 & 4640180 & 0.1 & 4135 & 505 & 0 & 150 & $100 \times$ & DWGSIM \\
ReadSet5 & E. coli-Mut1 & 4639675 & 0.1 & 4640 & 0 & 0 & 150 & $20 \times$ & DWGSIM \\
ReadSet6 & E. coli-Mut2 & 4639675 & 0.1 & 4640 & 0 & 0 & 150 & $20 \times$ & DWGSIM \\
ReadSet7 & E. coli-Mut3 & 4639138 & 0.1 & 4205 & 0 & 537 & 150 & $20 \times$ & DWGSIM \\
ReadSet8 & E. coli-Mut4 & 4640180 & 0.1 & 4135 & 505 & 0 & 150 & $20 \times$ & DWGSIM \\
ReadSet9 & E. coli-Mut1 & 4639675 & 0.1 & 4640 & 0 & 0 & 150 & $20 \times$ & ART \\
ReadSe10 & E. coli-Mut2 & 4639675 & 0.1 & 4640 & 0 & 0 & 150 & $20 \times$ & ART \\
ReadSet11 & E. coli-Mut3 & 4639138 & 0.1 & 4205 & 0 & 537 & 150 & $20 \times$ & ART \\
ReadSet12 & E. coli-Mut4 & 4640180 & 0.1 & 4135 & 505 & 0 & 150 & 20x & ART \\
\hline
\end{tabular}

extracted from the SAM file by a script and the consensus sequence is constructed by SAMtools.

\section{Evaluation metrics}

To evaluate EIM pipeline, we calculate some contiguity and quality metrics by QUAST [23] for contig sets (genomes) reconstructed by ExactMapping step, EIM and the mappers.

We use two metrics to compare the contiguity of the contig sets as follows:

- Contigs-500: The number of contigs with length of greater than $500 \mathrm{bp}$ belonging to the contig set.

- N50: The length of the smallest contig in the set that contains the fewest (largest) contigs whose combined length represents at least $50 \%$ of assembly [24].

We use quality metrics for indicating the accuracy of the reconstructed genomes. To calculate some quality metrics, each set of contigs is aligned to the target (or reference) genome to find the number of errors regarding to each contig set as below:

- Errors: The total number of mismatches and indels (insertions and deletions) in the aligned contigs relative to the target genome.

- IUPAC-codes: The total number of IUPAC ambiguity positions in the contig set.

- Genome-Fraction: The percentage of the target (or reference) genome covered by the aligned contigs.

when the target genome is not available, we apply the following quality measure to test the accuracy of the reconstructed genomes.

- Remapped-Reads: The percentage of the reads which are identically mapped (i.e. without mismatches and indels) onto the contigs.

\section{Results}

A set of reads and a reference genome are given to EIM pipeline as inputs and then EIM constructs a set of contigs as output by stepwise mapping of the reads onto the reference. The sequencing errors and genetic differences as well as repetitive regions in the genome are the factors which introduce mapping errors such as mismatches and indels into the contigs relative to the target genome.

To evaluate the results of EIM pipeline, we use different datasets in terms of similarity between the target and reference genomes as follows:

1. By considering a reference genome identical to the target genome, we initially assess our pipeline where the real read set from $E$. coli $K 12$ includes sequencing errors.

2. According to the high similarity between any human genome and the human reference, we investigate results of EIM pipeline where the real reads from a human chromosome 14 contain sequencing errors as well as SNVs. It is to be noted that the target genome is not available.

3. By simulating some target genomes highly similar to E. coli $\mathrm{K} 12$ genome, we examine EIM pipeline in which the simulated reads include SNVs. In this way, we can test the accuracy of EIM more precisely since the target genomes are available.

4. By using a closely related genome to E. coli $K 12$ as a reference, we perform EIM pipeline on a real read set from $E$. coli $K 12$ to assess our pipeline where the similarity between the target and reference genomes is not very high.

For completing the evaluation of EIM, we apply different mappers on a real read set from E. coli $K 12$ and a closely related genome to it as a reference, and then compare the 
results of EIM pipeline to the respective mappers. In addition, we evaluate our pipeline on eukaryotic genomes of human and Arabidopsis thaliana.

\section{Assessment of EIM on a real dataset of E. coli K12}

To test the accuracy of EIM, we examine the effect of sequencing errors without considering any other factors. For this purpose, E. coli K12 genome and its reads generated by using Illumina are given to EIM as inputs. Accordingly, the target and reference genomes are the same and the read set includes sequencing errors.

An Illumina sequencer has an error rate of $<0.1 \%$ [25], because of which only $61.79 \%$ of the reads can be mapped at the first step of EIM pipeline (ExactMapping). However, contigs constructed from the uniquely mapped reads cover nearly entire of the target genome $(99.995 \%$ in Table 3). At the second step of our pipeline (InExactMapping), remaining reads from the first step are mapped onto just $0.005 \%$ of the reference. As shown in Table 3, the last step of EIM (MergingContigs) produces a contiguous contig including 2 errors, while Bowtie 2 mapper makes 11 contigs containing the same number of errors on this sample data. Although Bowtie2 generates more contigs than EIM, the Genome-Fraction values of both contig sets are the same (100\%) because the gaps between contigs of Bowtie 2 are too small compared to the total length of the target genome.

This assessment shows that contig sets reconstructed by EIM and Bowite2 are the same in terms of accuracy when the read set contains sequencing errors.

\section{Assessment of EIM on a real dataset of human}

\section{chromosome 14}

In this assessment, our goal is to investigate the accuracy of EIM where the set of reads extracted from a genome

Table 3 Real datasets analysis where the inputs of EIM pipeline are the read set and reference genome

\begin{tabular}{llll}
\hline Assembly & Exact & EIM & Bowtie2 \\
\hline E. coli & & & \\
Contigs-500 & 64 & 1 & 11 \\
N50 (kbp) & 1250.9 & 4639.673 & 597.8 \\
Errors & 0 & 2 & 2 \\
Genome-Fraction (\%) & 99.995 & 100 & 100 \\
Human chromosome 14 & & & \\
Contigs-500 & 27312 & 451 & 1179 \\
N50 (kbp) & 6 & 407.6 & 174.1 \\
Errors & 18 & 44407 & 44911 \\
Genome-Fraction (\%) & 93.22 & 99.80 & 99.71 \\
\hline
\end{tabular}

The evaluation metrics has been defined in the text. The columns headed 'Exact', 'EIM' and 'Bowtie2' represent the contiguity and quality of contigs constructed by ExactMapping step of EIM, EIM and Bowtie2, respectively includes sequencing errors as well as SNVs and indels relative to the reference. We perform EIM on the human chromosome 14 reference and the reads from a human chromosome 14.

Due to sequencing errors and genetic differences between human genomes, only about half of reads (58.33\%) are aligned at the ExactMapping step. The contigs constructed from this volume of the reads cover 93.22\% of the chromosome 14 reference (Table 3). Furthermore, the results presented in Table 3 show that EIM makes significantly fewer contigs than Bowtie2. In other words, the comparison of N50 values indicates that EIM can make a contig set more contiguous than that of Bowtie2. Moreover, the contigs of EIM include fewer errors relative to the reference than those of Bowtie2. Although comparing with the reference genome gives insight into the error rate of the reconstructed genomes, some differences are true differences rather than errors. Since the target genome is not available, we use the read set to assess the accuracy of EIM. In this way, the reads are mapped without mismatches and indels to the reconstructed genomes to calculate Remapped-Reads values. The results of the remapping show that the RemappedReads values for the genomes reconstructed by EIM and Bowtie 2 are $60.87 \%$ and $58.68 \%$ respectively. This is an appropriate evidence that the reconstructed genome by EIM is more accurate than that of Bowtie2.

Our results show that when the target and reference genomes are highly similar, EIM pipeline can reconstruct a more accurate genome than the one rebuilt by Bowtie2 mapper.

\section{Assessment of EIM on simulated data}

To assess the accuracy of EIM more precisely, the target genome sequences are required. Since target sequences are typically not available for most of individuals and strains, we use simulated data. To do so, we make some genome sequences derived from $E$. coli $\mathrm{K} 12$ genome by creating mismatches and indels using different rates and then simulate read sets from the mutated genomes (Table 2).

We test EIM pipeline on ReadSet1 (Table 2) and E. coli $K 12$ as a reference genome. To compare contigs generated by EIM and Bowtie2, we align both contig sets against E. coli-Mut1 (the target genome) and present the results in the second, third and last columns of Table 4. Although EIM pipeline rebuilds a contiguous contig, it introduces more errors than Bowtie2. It is also worth mentioning that the contigs of ExactMapping step of EIM called Exact contigs have $90.285 \%$ Genome-Fraction value which in comparison with that obtained by real data experiment (99.995\% in Table 3) is very low. It seems that a lower Genome-Fraction value of Exact contigs leads to the higher errors in the final contigs produced by EIM. 
Table 4 Simulated ReadSet1 analysis where the inputs of EIM pipeline are the read set and either the reference genome (ref) or the genome reconstructed by Bowtie2 (cns-bt)

\begin{tabular}{lllllll}
\hline c1 1 & c2 & c3 & c4 & c5 & c6 & c7 \\
\hline Assembly & Exact & EIM & Exact & EIM (v1) & EIM (v2) & Bowtie2 \\
& /ref & /ref & /cns-bt $/ c n s-b t$ & /cns-bt & / ref \\
Contigs-500 & 2817 & 1 & 68 & 9 & 2 & 6 \\
N50 (kbp) & 1.82 & 4639.6 & 156.4 & 1108.9 & 3543.3 & 2385.650 \\
Errors & 3 & 45 & 0 & 28 & 29 & 38 \\
Genome-Fraction (\%) & 90.285 & 100 & 99.381 & 99.992 & 99.999 & 100
\end{tabular}

The evaluation metrics has been defined in the text. The columns headed 'Exact/ref', 'EIM/ref', 'EIM (v1)/cns-bt', 'EIM ( $v 2)$ /cns-bt' and 'Bowtie2' represent the contiguity and quality of the respective contigs. Also E. coli K12 genome is denoted by 'ref' and the consensus sequence constructed by Bowtie 2 on E. coli genome is denoted by 'cns-bt'

We need to point out that the more fraction of the target genome is covered by Exact contigs, the smaller parts of the reference remain for InExactMapping step of EIM. Hence the probability that the leftover reads are aligned at true locations is increased and as a result, the error rate of the reconstructed genome is reduced. Furthermore, the fraction of the target genome covered by Exact contigs is directly proportional to the similarity between the target and reference genomes. In other words, the higher similarity between the target and reference genomes leads to fewer errors in the genome reconstructed by EIM pipeline. Accordingly, since the genome sequence reconstructed by a mapper is more similar to the target genome than to the reference (ref), the genome sequence reconstructed by Bowtie2 (cns-bt) is fed to EIM instead of the reference as input.

The results can be seen in the fourth and fifth columns of Table 4. The comparison of the second and fourth columns shows that by giving the genome sequence reconstructed by Bowtie 2 instead of the reference sequence to EIM as the input, the Genome-Fraction value of Exact contigs increases from 90 to $>99 \%$. In addition, the number of errors in final contigs of EIM decreases from 45 to 28 . It suggests that the genome sequence reconstructed by a mapper is a better input for our pipeline as it leads to a lower error rate. Our analysis up to this point shows that by feeding $c n s-b t$ instead of ref to EIM pipeline as input, the error rate is reduced. It is important to note that the error rate decreasing is valuable only when EIM rather maintains the same N50 and Genome-Fraction values as those of the input genome. However, the results of EIM in the fifth column compared to the last column of Table 4 indicate that this condition is not satisfied.

We observed that cns-bt includes 137 IUPAC-codes while ref contains no IUPAC-codes. Furthermore, the genome reconstructed by mapping a read set onto a reference sequence containing IUPAC-codes is less contiguous than the reference because SAMtools makes a consensus sequence including ' $\mathrm{N}$ ' in the IUPAC-code positions. Thus the existence of IUPAC-codes in the input genome of EIM yields a more fragmented genome as output. To solve this issue, we execute SAMtools with a parameter allowing to build consensus in the IUPAC-code positions instead of substituting ' $\mathrm{N}$ ' ambiguity character ("Tools" subsection). As shown in the sixth column of Table 4, EIM with this modification makes contigs which in addition to including less errors than $c n s-b t$ (the input genome), are nearly as contiguous as cns-bt and with high coverage of the target genome. In the following, EIM described in the fifth and sixth columns of Table 4 are considered as versions one $(v 1)$ and two $(v 2)$, respectively.

Tables 5 and 6 represent the results of applying EIM (v2) pipeline and Bowtie2 mapper to the simulated read

Table 5 Simulated high coverage datasets analysis where the inputs of EIM pipeline are the read set and genome reconstructed by Bowtie2

\begin{tabular}{|c|c|c|c|}
\hline Assembly & Exact & $\operatorname{EIM}(v 2)$ & Bowtie2 \\
\hline \multicolumn{4}{|l|}{ ReadSet1 } \\
\hline Contigs-500 & 68 & 2 & 6 \\
\hline N50 (kbp) & 156.4 & 3543.3 & 2385.6 \\
\hline Errors & 0 & 29 & 38 \\
\hline IUPAC-codes & 0 & 11 & 137 \\
\hline Genome-Fraction (\%) & 99.381 & 99.999 & 100 \\
\hline \multicolumn{4}{|l|}{ ReadSet2 } \\
\hline Contigs-500 & 142 & 3 & 5 \\
\hline N50 (kbp) & 62.7 & 1371.6 & 939.1 \\
\hline Errors & 0 & 45 & 92 \\
\hline IUPAC-codes & 0 & 29 & 246 \\
\hline Genome-Fraction (\%) & 99.338 & 100 & 99.997 \\
\hline \multicolumn{4}{|l|}{ ReadSet3 } \\
\hline Contigs-500 & 96 & 6 & 3 \\
\hline N50 (kbp) & 94.4 & 1096.2 & 3267.7 \\
\hline Errors & 2 & 54 & 87 \\
\hline IUPAC-codes & 0 & 11 & 140 \\
\hline Genome-Fraction (\%) & 99.285 & 99.997 & 100 \\
\hline \multicolumn{4}{|l|}{ ReadSet4 } \\
\hline Contigs -500 & 77 & 3 & 3 \\
\hline N50 (kbp) & 115.5 & 2337.4 & 1530.2 \\
\hline Errors & 6 & 55 & 72 \\
\hline IUPAC-codes & 0 & 15 & 34 \\
\hline Genome-Fraction (\%) & 99.436 & 99.998 & 100 \\
\hline
\end{tabular}

The evaluation metrics has been defined in the text. The columns headed 'Exact', 'EIM (v2)' and 'Bowtie2' represent the contiguity and quality of contigs constructed by ExactMapping step of EIM, EIM (v2) and Bowtie2, respectively 
Table 6 Simulated low coverage datasets analysis where the inputs of EIM pipeline are the read set and genome reconstructed by Bowtie2

\begin{tabular}{|c|c|c|c|c|c|c|c|}
\hline Assembly & Exact & $\operatorname{EIM}(v 2)$ & Bowtie2 & & Exact & $\operatorname{EIM}(v 2)$ & Bowtie2 \\
\hline \multicolumn{4}{|l|}{ DWGSIM simulator } & \multicolumn{4}{|l|}{ ART simulator } \\
\hline ReadSet5 & & & & ReadSet9 & & & \\
\hline Contigs-500 & 172 & 14 & 58 & & 137 & 13 & 56 \\
\hline N50 (kbp) & 43.9 & 735.7 & 159.6 & & 75.2 & 909.5 & 175.3 \\
\hline Errors & 0 & 53 & 64 & & 1 & 45 & 53 \\
\hline IUPAC-codes & 0 & 36 & 137 & & 0 & 38 & 102 \\
\hline Genome-Fraction (\%) & 98.899 & 99.991 & 99.983 & & 98.912 & 99.983 & 99.965 \\
\hline ReadSet6 & & & & ReadSet10 & & & \\
\hline Contigs-500 & 163 & 6 & 64 & & 178 & 18 & 63 \\
\hline N50 (kbp) & 44.4 & 1698.5 & 112.2 & & 45.4 & 485.2 & 116.9 \\
\hline Errors & 1 & 68 & 92 & & 2 & 68 & 83 \\
\hline IUPAC-codes & 0 & 28 & 95 & & 0 & 39 & 187 \\
\hline Genome-Fraction (\%) & 98.843 & 99.998 & 99.976 & & 98.871 & 99.988 & 99.984 \\
\hline ReadSet7 & & & & ReadSet11 & & & \\
\hline Contigs-500 & 424 & 11 & 55 & & 425 & 17 & 70 \\
\hline N50 (kbp) & 16.6 & 590.9 & 125.9 & & 18 & 386.6 & 115.4 \\
\hline Errors & 2 & 185 & 361 & & 6 & 179 & 369 \\
\hline IUPAC-codes & 0 & 24 & 117 & & 0 & 38 & 151 \\
\hline Genome-Fraction (\%) & 98.666 & 99.993 & 99.985 & & 98.697 & 99.989 & 99.983 \\
\hline ReadSet8 & & & & ReadSet12 & & & \\
\hline Contigs-500 & 397 & 17 & 56 & & 366 & 13 & 49 \\
\hline N50 (kbp) & 18.9 & 493.4 & 141.1 & & 21.6 & 529.5 & 127.6 \\
\hline Errors & 8 & 190 & 331 & & 8 & 186 & 322 \\
\hline IUPAC-codes & 0 & 21 & 105 & & 0 & 23 & 121 \\
\hline Genome-Fraction (\%) & 98.771 & 99.99 & 99.979 & & 98.799 & 99.983 & 99.982 \\
\hline
\end{tabular}

The evaluation metrics has been defined in the text. The columns headed 'Exact', 'EIM (v2)' and 'Bowtie2' represent the contiguity and quality of contigs constructed by ExactMapping step of EIM, EIM (v2) and Bowtie2, respectively. The results of running the pipeline on datasets simulated by DWGSIM and ART are shown in left and right side of the table, respectively

sets with high and low coverage, respectively. As illustrated by the results, not only can EIM ( $v 2)$ decrease the error and IUPAC-code rates, but it can also maintain the contiguity and Genome-Fraction value very close to Bowtie2.

The results of this assessment show that our pipeline can improve the genome sequence reconstructed by Bowtie2 mapper in terms of accuracy when a highly similar reference to the target genome is available and the read set includes SNVs relative to the reference.

\section{Assessment of EIM on a real dataset of E. coli K12 and a closely related genome}

In this assessment, we examine the accuracy of EIM when similarity between the target and reference genomes is not so high. The application is where a reference is not available and a closely related genome is used as a reference. We apply E. coli O145:H28 as a closely related genome to E. coli K12.

To evaluate EIM on the read set from E. coli K12, a genome sequence is reconstructed from mapping the reads onto E. coli O145:H28 genome by Bowtie2, then the reconstructed genome and the reads are given to EIM as inputs. Table 7 shows that the contig sets generated by EIM ( $v 1)$ and EIM $(v 2)$ contain fewer errors and IUPACcodes than that of Bowtie2. Moreover, EIM ( $v 2$ ) can make contigs which have nearly the same Genome-Fraction value and N50 size as those of Bowtie2.

It should be noticed that the Genome-Fraction values of the contigs produced by EIM and Bowtie2 are less than $90 \%$. In such cases where there is no reference available and the related genome is not highly similar to the target 
Table 7 Real dataset analysis where a closely related genome is used as a reference

\begin{tabular}{|c|c|c|c|c|c|c|}
\hline Assembly & Exact & $\operatorname{EIM}(v 1)$ & $\operatorname{EIM}(v 2)$ & Bowtie2 & MaSuRCA & EIM $(v 1)+$ MaSuRCA \\
\hline Contigs-500 & 497 & 334 & 263 & 259 & 114 & 114 \\
\hline N50 (kbp) & 12.3 & 22.2 & 32 & 29.3 & 106 & 106 \\
\hline Errors & 58 & 618 & 1190 & 2472 & 2407 & 1786 \\
\hline IUPAC-codes & 0 & 17 & 56 & 280 & 0 & 5 \\
\hline Genome-Fraction (\%) & 87.013 & 87.811 & 88.578 & 88.575 & 99.058 & 99.058 \\
\hline
\end{tabular}

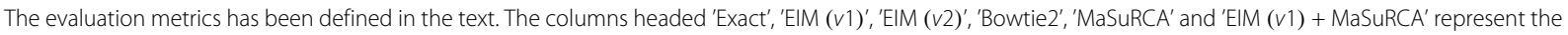
contiguity and quality of contigs constructed by ExactMapping step of EIM, EIM ( $v 1)$, EIM ( $v 2)$, Bowtie2, MaSuRCA and combining the contig sets of EIM ( $v 1)$ and MaSuRCA assembler, respectively

genome, de novo genome assembly is a better approach for reconstructing the genome sequence. However, the genome sequences generated by de novo assemblers are not error-free. For this reason, approaches for improving the accuracy of de novo assembled contigs are needed. Here we use the contigs generated by EIM to improve the contigs produced by a de novo assembler. In fact, we use version one of EIM pipeline because contigs of EIM ( $v 1)$ include less errors than those of EIM ( $v 2)$. The read set is assembled by MaSuRCA [26], one of the best assemblers at GAGE-B [27], then the contigs constructed by EIM and MaSuRCA are combined into a contig set including fewer errors than the contigs of MaSuRCA (Table 7).

This analysis indicates that when a closely related genome is used as a reference, and thus the reference and target genomes are not highly similar, EIM ( $v 2)$ can reconstruct a genome sequence with the same contiguity and Genome-Fraction value including less errors and IUPACcodes than the genome reconstructed by Bowtie2 mapper. In addition, the genome rebuilt by EIM $(v 1)$ can decrease the error rate of a genome sequence generated by a de novo assembler such as MaSuRCA.

\section{Evaluation of EIM by different mappers}

To evaluate the performance of our pipeline by using mappers other than Bowtie2, we select BWA as a popular and widely used mapper and Yara as one of the state-ofthe-art mappers. We use the three mappers and version 2 of EIM on the read set from E. coli K12 and E. coli O145:H28 genome as a reference. For each mapper, the genome reconstructed by the mapper is given to EIM ( $v 2)$ as input and the mapper itself is applied for aligning reads in the second step of EIM ( $v 2$ ) (i. e. InExactMapping).

As illustrated in Fig. 2, for all mappers, EIM pipeline maintains N50 size and Genome-Fraction value close but not identical to those of the mappers (Fig. 2a and b). It also reduces the number of errors and significantly decreases the number of IUPAC-codes (Fig. 2c and d).

Figure $2 \mathrm{e}$ shows the running times of the three mappers compared to EIM. Since the input genome of EIM is built by a mapper, the running time of reconstructing a genome by EIM is the total of mapper and EIM pipeline runtimes. In addition, the running time of reconstructing a genome by a mapper is the total of read mapping and consensus constructing runtimes, which the second one is more time-consuming. Our pipeline decreases the computational time of making a consensus by a two-step mapping. In ExactMapping, most of the reads are exactly aligned and a SAM file is made from which the consensus sequence can be constructed by a simple and fast script without using SAMtools. Moreover, only a low percentage of reads is transferred to InExactMapping step and thus the consensus sequence is made rapidly by SAMtools in this stage. Consequently, the overhead time of reconstructing the E. coli genome by EIM pipeline after running a mapper is less than one-third of that of the respective mapper (Fig. 2e).

This evaluation demonstrates that EIM pipeline can be used as a post-processing tool to improve the genome reconstructed by a mapper to a more accurate one in an acceptable runtime while maintaining the contiguity and Genome-Fraction value of the input genome.

\section{Evaluation of EIM on de novo assembled genomes}

In this section, we assess the effect of EIM pipeline on the results of de novo assemblies. For this purpose, we compare EIM with Pilon framework [28] and Columbus module of Velvet assembler [29]. These tools get a draft or reference genome and mapped reads on it, to apply read mappings for improving genome assembly.

In the following, we first generate two genomes by Velvet and MaSuRCA assemblers on the real read set from E. coli K12. Then each draft genome is inputted to EIM, Pilon, and Columbus.

As illustrated in Fig. 3, all frameworks reduce the number of errors and dramatically decrease the number of IUPAC-codes when that of the draft genome is too high (Fig. 3a and b). Although EIM and Columbus decrease N50 size (Fig. 3c), they maintain Genome-Fraction value close to those of draft genomes (Fig. 3d).

The results of this comparison show that EIM pipeline has an impact on reducing the error rate of the genomes generated by de novo assembly. 

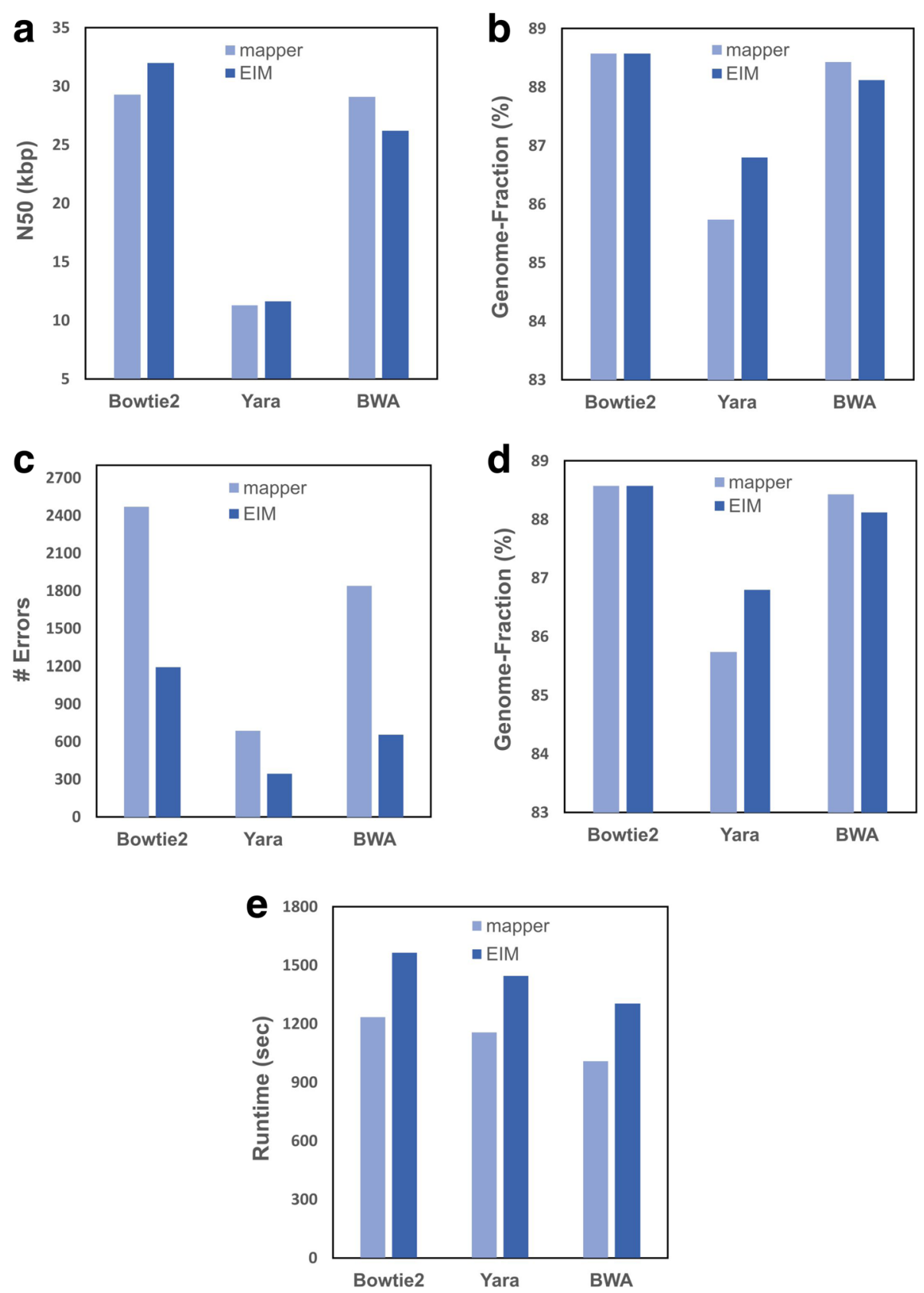

Fig. 2 The comparison of contigs generated by Bowtie2, Yara and BWA with the respective contigs of EIM on the real read set of E. coli K12. Firstly, the mappers were executed on the read set and the reference, and then the contig sets were generated. Secondly, for each mapper, EIM ( $v 2$ ) was run on the read set and the contig set constructed by the mapper while using it at the second step for mapping. Finally, the contiguity and quality of contigs were computed as a N50 size $\mathbf{b}$ Genome-Fraction value $\mathbf{c}$ The number of errors $\mathbf{d}$ The number of IUPAC codes. In addition, the running time of obtaining contigs was measured and showed in seconds (e)

\section{Evaluation of EIM on eukaryotic genomes}

For the final evaluation, we run EIM pipeline on the datasets of human as a mammalian and Arabidopsis thaliana as a model plant. To evaluate EIM on human, we select the smallest and the largest chromosomes as well as a chromosome with average length namely Chr21, Chr1, and Chr10, respectively and extract the reads of each one from real samples of the whole human genome. Then we run EIM on each dataset separately. For evaluating our pipeline on Arabidopsis thaliana, we simulate a dataset for all chromosomes of bur-0 strain and use TAIR10 as the reference to run EIM. 


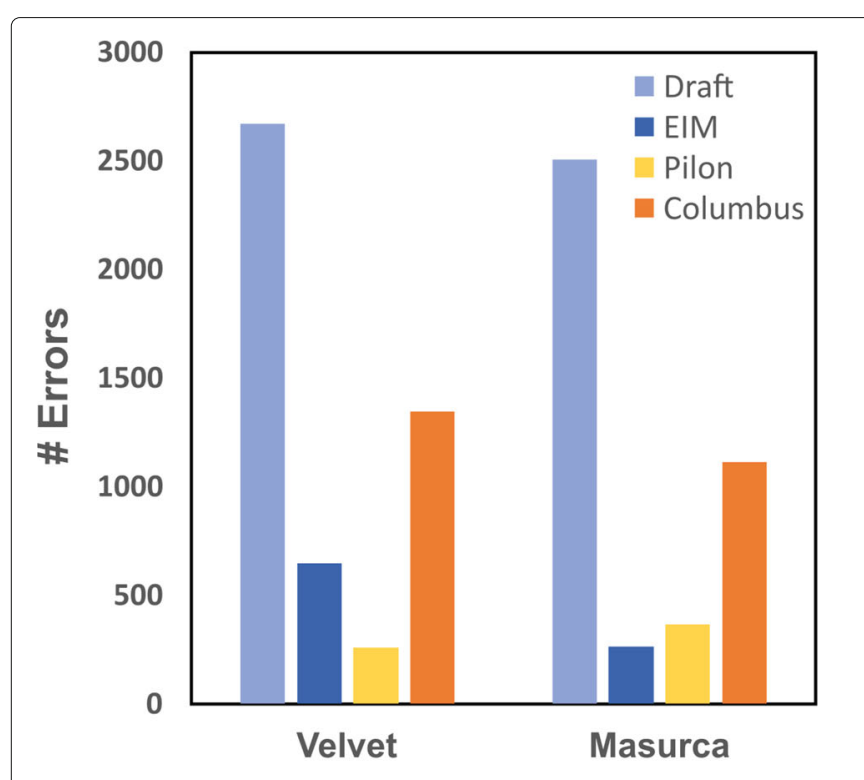

(a)

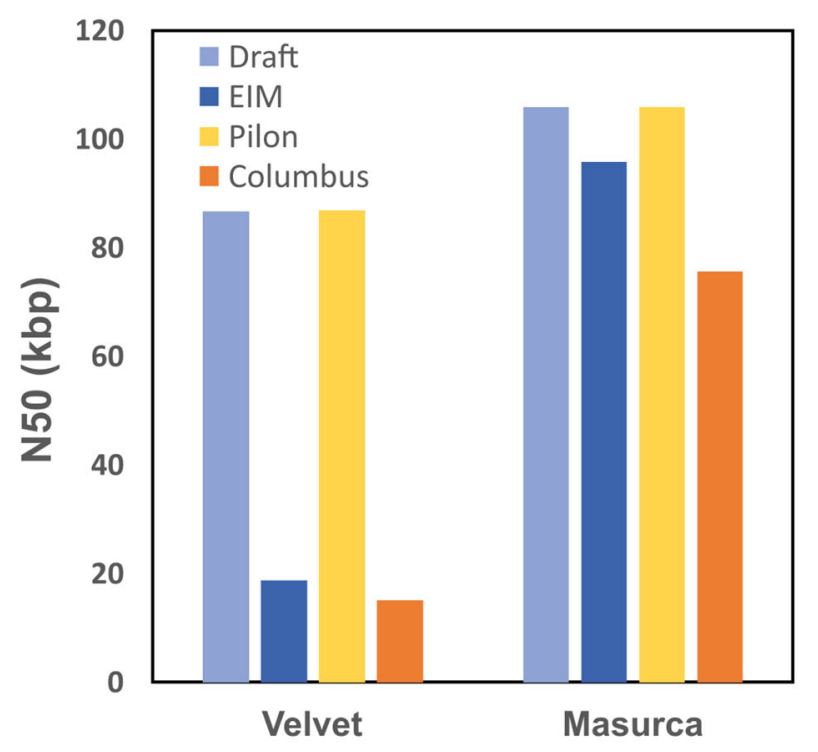

(c)

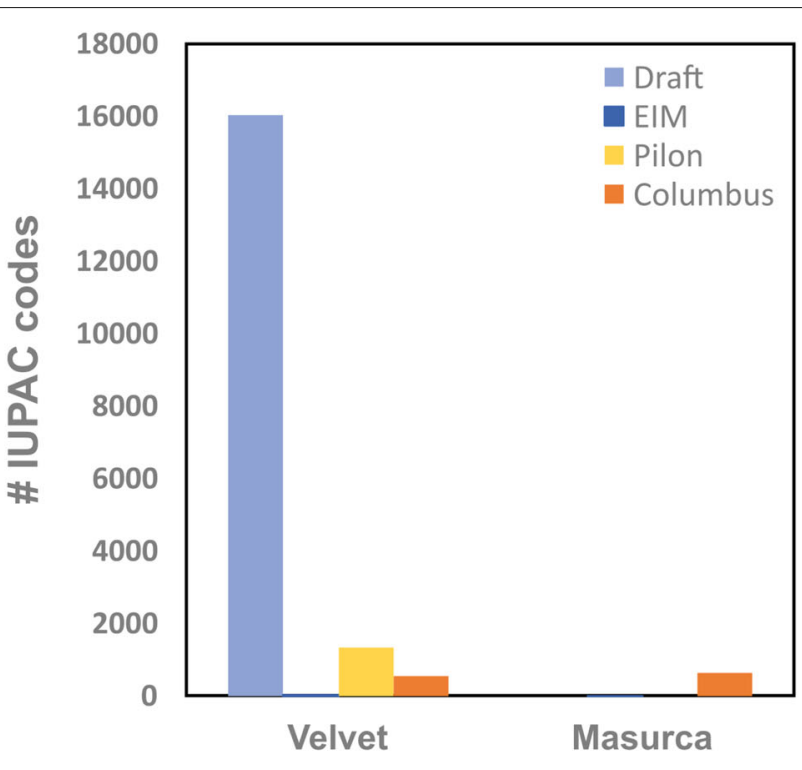

(b)

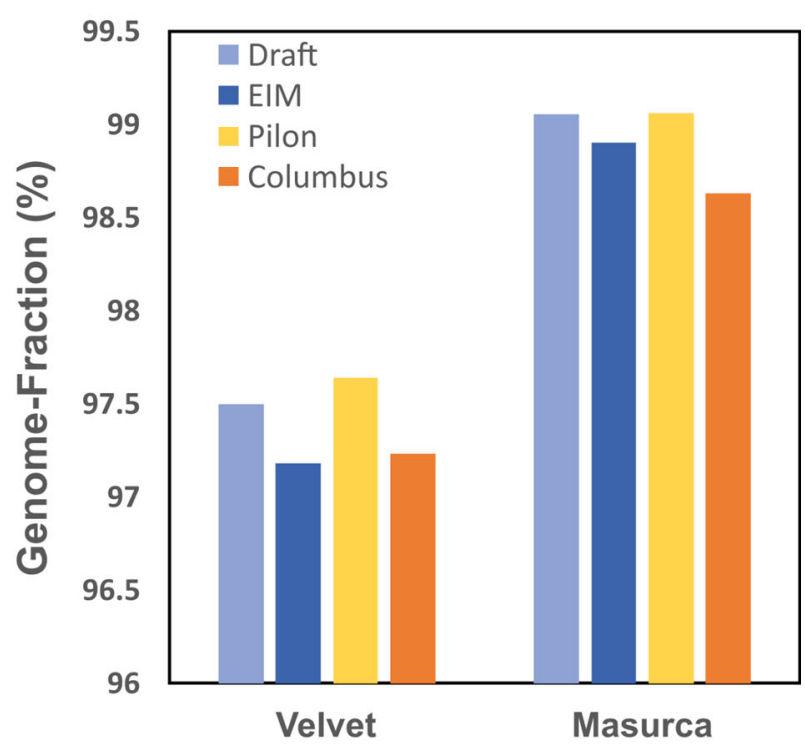

(d)

Fig. 3 The comparison of contigs generated by EIM, Pilon and Columbus on the real read set of E. coli K12.Firstly, two draft genomes were generated by Velvet and MaSuRCA de novo assemblers. Secondly, EIM, Pilon and Columbus were run by each draft and mapped reads on the draft. Finally, the quality and contiguity of contigs were computed as a The number of errors $\mathbf{b}$ The number of IUPAC codes $\mathbf{c}$ N50 size $\mathbf{d}$ Genome-Fraction value

As shown in Table 8, EIM pipeline reduces error rates on all three human chromosomes and bur- 0 strain of Arabidopsis. To be precisely measured the accuracy of generated contigs, the reads are exactly mapped onto each contig set to calculate Remapped-Reads value. As seen, EIM increases Remapped-Reads values. Furthermore, the results show that our pipeline considerably increases the N50 size of contig sets generated for human chromosomes because of the high similarity between human genomes.
In order to examine the effect of different chromosomal regions on accuracy of EIM, we test our pipeline on portions of a human chromosome. To achieve this goal, we divide Chr1, the largest human chromosome, to twenty five same-length regions as follows:

$$
P=\left\{p_{1}, \ldots, p_{25}\right\} \text { for each } i\left|p_{i}\right| \simeq 10 \mathrm{Mbp} \text {. }
$$

The number of ambiguity characters (Ns) is assessed in each $p_{i} 1 \leq i \leq 25$ (Fig. 4). We omit $p_{14}$ because this region 
Table 8 Evaluating EIM on some eukaryotic datasets

\begin{tabular}{|c|c|c|}
\hline Assembly & $\operatorname{EIM}(v 2)$ & Bowtie2 \\
\hline \multicolumn{3}{|l|}{ Human chromosome 1} \\
\hline Contigs-500 & 2497 & 5018 \\
\hline N50 (kbp) & 420.7 & 158.2 \\
\hline Errors & 115381 & 120726 \\
\hline IUPAC-codes & 7862 & 158247 \\
\hline Genome-Fraction (\%) & 99.828 & 99.614 \\
\hline Remapped-Reads (\%) & 52.02 & 50.95 \\
\hline \multicolumn{3}{|l|}{ Human chromosome 10} \\
\hline Contigs-500 & 1443 & 2478 \\
\hline N50 (kbp) & 399.9 & 149.2 \\
\hline Errors & 70478 & 73842 \\
\hline IUPAC-codes & 5508 & 112333 \\
\hline Genome-Fraction (\%) & 99.209 & 99.034 \\
\hline Remapped-Reads (\%) & 51.72 & 49.93 \\
\hline \multicolumn{3}{|l|}{ Human chromosome 21} \\
\hline Contigs-500 & 1239 & 2362 \\
\hline N50 (kbp) & 237.8 & 101 \\
\hline Errors & 22904 & 23579 \\
\hline IUPAC-codes & 3232 & 46155 \\
\hline Genome-Fraction (\%) & 99.114 & 97.73 \\
\hline Remapped-Reads (\%) & 44.58 & 42.23 \\
\hline \multicolumn{3}{|c|}{ Arabidopsis Thaliana (bur-0) } \\
\hline Contigs-500 & 6936 & 6987 \\
\hline N50 (kbp) & 428.8 & 417.4 \\
\hline Errors & 136539 & 179312 \\
\hline IUPAC-codes & 4842 & 2370 \\
\hline Genome-Fraction (\%) & 98.634 & 98.572 \\
\hline Remapped-Reads (\%) & 66.32 & 65.24 \\
\hline
\end{tabular}

The evaluation metrics has been defined in the text. The columns headed 'EIM ( $v 2)^{\prime}$ and 'Bowtie2' represent the contiguity and quality of contigs obtained based on the results of EIM ( $(2)$ and Bowtie2, respectively

is a whole sequence of Ns. We then run EIM on the read set of Chr 1 and each $p_{i} 1 \leq i \leq 25$ andi $\neq 14$, separately.

As shown in Fig. 5, EIM pipeline increases N50 values and reduces error numbers, and significantly decreases IUPAC numbers for all regions. Note that, because of the high fraction of Ns in centromere region, contigs generated by Bowtie 2 and EIM on $p_{13}$ and $p_{15}$ have low N50 size and low error numbers (Fig. 5a and d).

In addition, EIM increases Remapped-Reads values for all regions except for the first one (Fig. 5b). To explore the reason, we break the $p_{1}$ region from Ns and select two of five yielded portions called $p_{1 \_1}(\sim 2.1 \mathrm{Mbp})$ and $p_{1 \_2}$ $(\sim 7.2 \mathrm{Mbp})$ for analysis because their length is more than $1 \mathrm{Mbp}$. Then we run EIM on the read set of Chr1 and

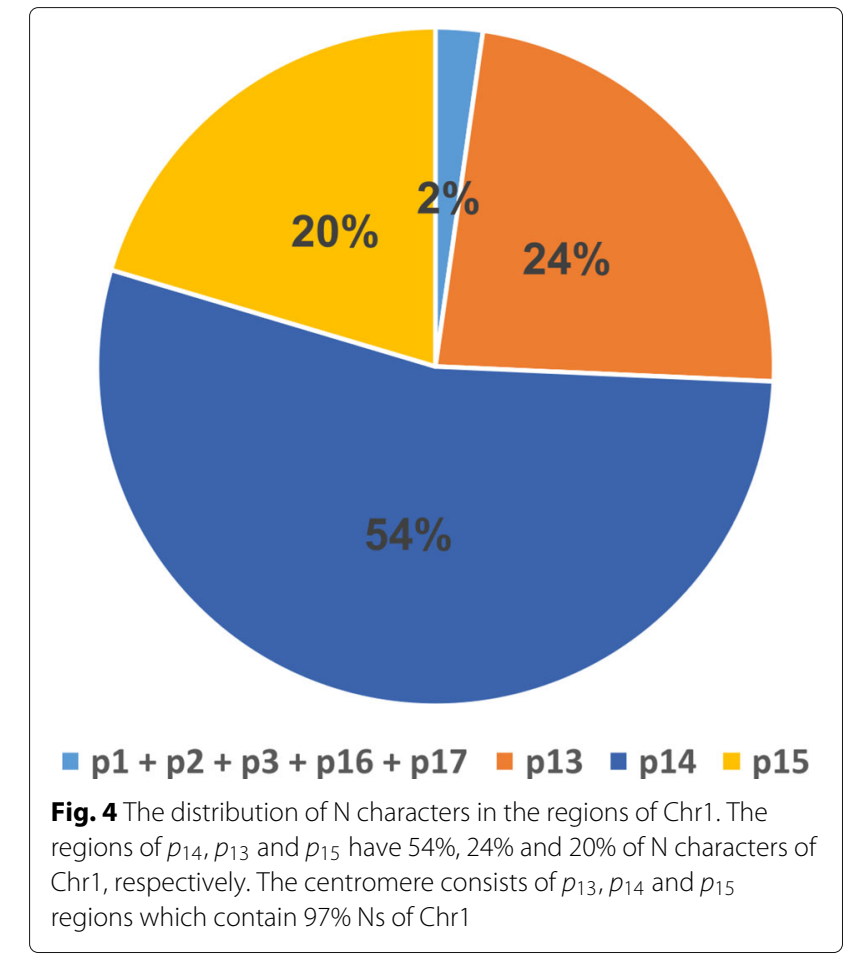

$p_{1 \_1}$ and $p_{1 \_2}$ regions, separately. The results show that the Remapped-Reads value of contigs generated by EIM is $0.05 \%$ more than that of Bowtie 2 for $p_{1 \_} 2$ while this value is $0.35 \%$ less than that of Bowtie 2 for $p_{1 \_} 1$. Thus the shorter portion i.e. $p_{1 \_1}$ leads to decreasing of the RemappedReads value of $p_{1}$ region. According to this observation, we examine GC-content of all regions of Chr1. The GCcontent of $p_{1 \_1}$ is $56 \%$ while GC-content of $p_{1 \_}$and other regions are less than $50 \%$ (Fig. 6).

The results from GC-content analysis suggest that running EIM on genomic regions with less than 50\% GCcontent can generate contigs which are more accurate than those of a mapper.

\section{Discussion}

As mentioned in the "Background" section, one of the most challenging aspects of genome sequence reconstruction from NGS data is the existence of multi-mapping reads. We claim that EIM pipeline decreases the number of multi-mapping reads and thus reduces the error rate of the reconstructed genome. To demonstrate this claim, we analyse each step of EIM separately. Let the input genome sequence of EIM be the genome reconstructed by a mapper like Bowtie2.

At ExactMapping step, the consensus sequence is built from the reads uniquely mapped and thus the resulting Exact contigs contain very low errors (see the 'Exact' column in Tables 6 and 7). Therefore the number of errors in the contigs of the next step plays a determining role in the error rate of the genome reconstructed by EIM. The 


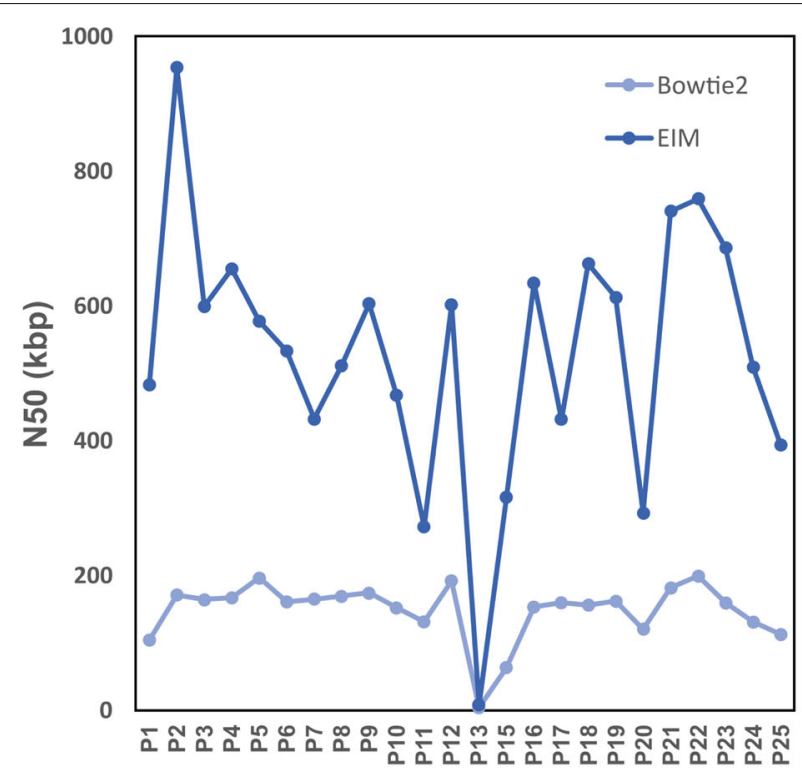

(a)

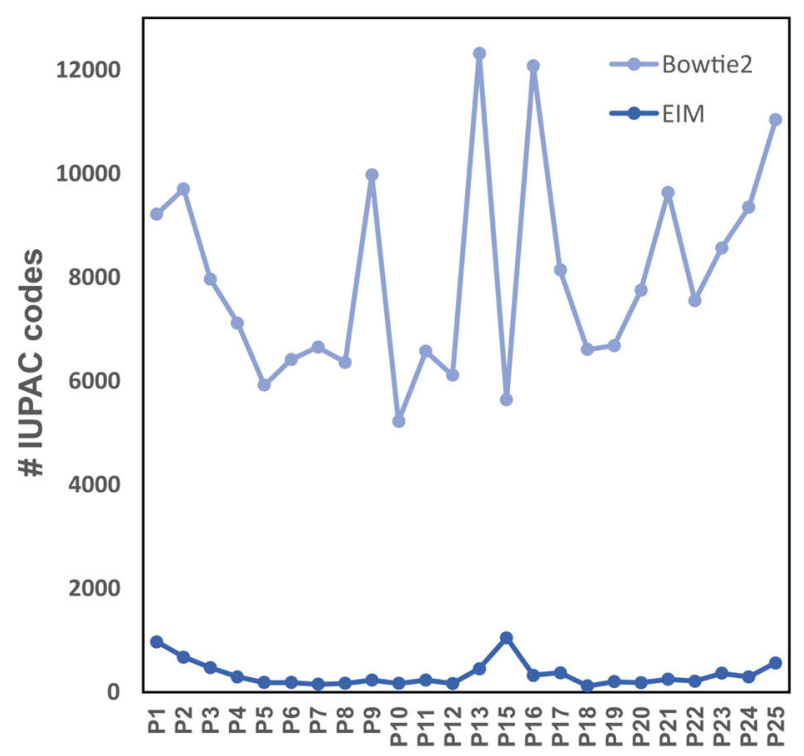

(c)

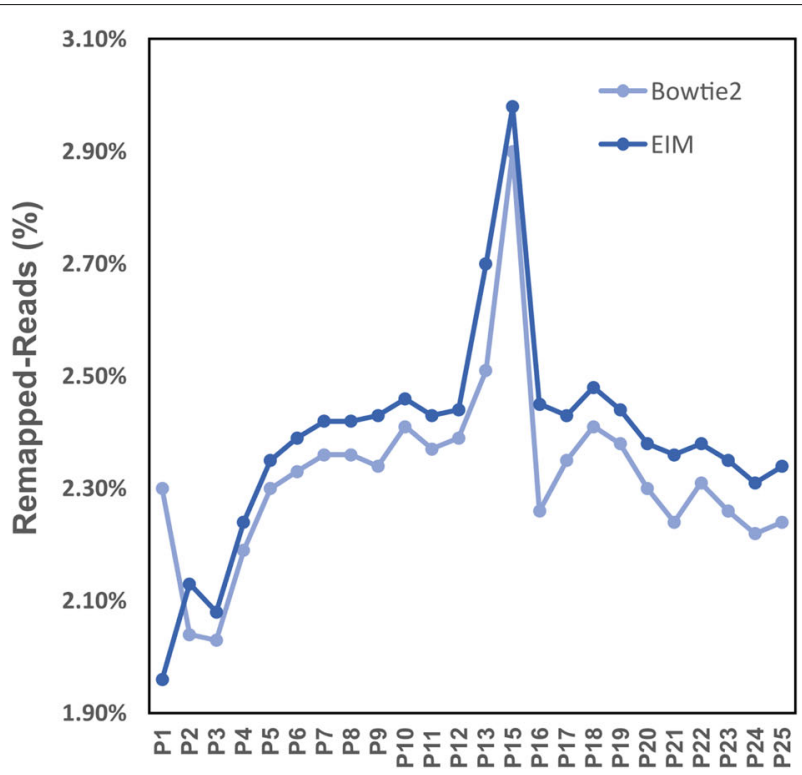

(b)

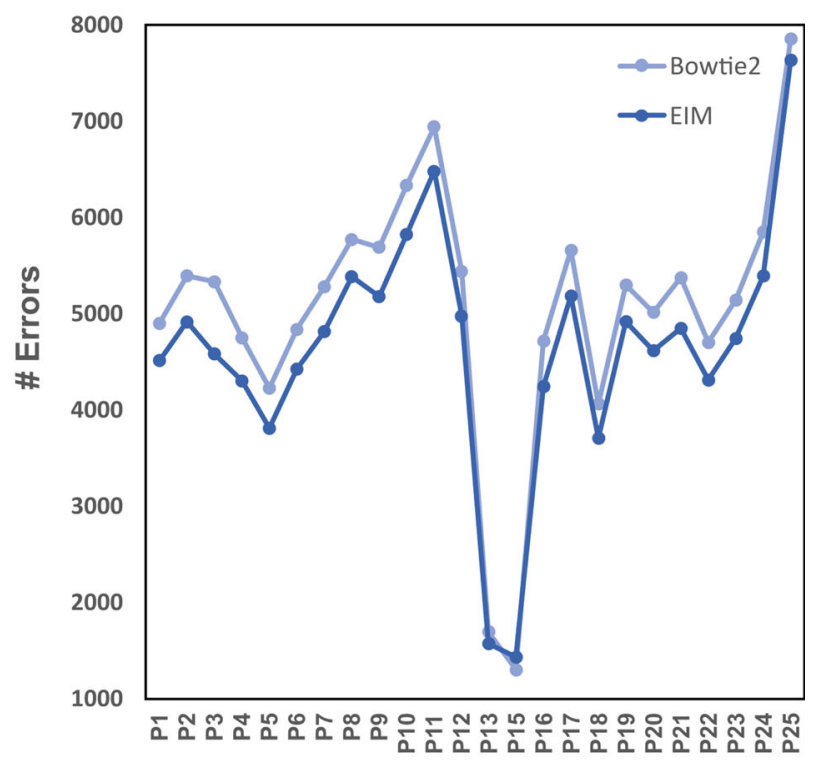

(d)

Fig. 5 The comparison of contigs generated by Bowtie2 and EIM on the regions of Chr1. Firstly, Chr1 was divided to some regions, $p_{i} 1 \leq i \leq 25$. Secondly, Bowtie2 and EIM were run by the read set of Chr1 and each $p_{i} 1 \leq i \leq 25 a n d i \neq 14$, separately. Finally, the quality and contiguity of contigs were computed as a N50 size $\mathbf{b}$ The Remapped-Reads value $\mathbf{c}$ The number of IUPAC codes $\mathbf{d}$ The number of errors

reads not applied in this step, namely multi-mapping and unmapped reads are transferred to the second step to be aligned with mismatches and indels.

At InExactMapping step, the remaining reads are aligned to the parts of the input genome not covered by any Exact contigs and then the consensus sequence is generated. To examine the effect of EIM on multi-mapping reads, we should compare the number of multi-mapping reads in this step to that obtained by mapping the reads onto the whole input genome. To do so, the reads that can be mapped at the second step of EIM, are aligned again to the whole input genome. Figure 7 shows that our pipeline leads to less multi-mapping reads on the simulated and real datasets. In fact, on the simulated datasets, EIM can decrease the number of multi-mapping reads by finding unique mapping locations for $17 \%$ of them on average. 


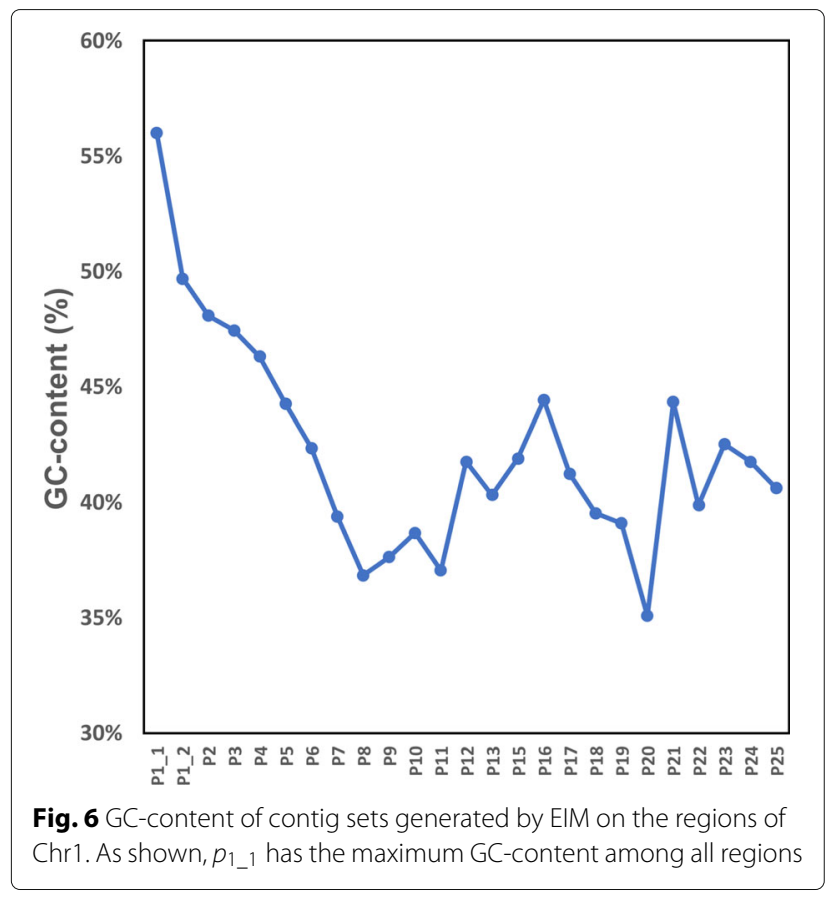

To complete the examination of the effect of EIM pipeline on multi-mapping reads, EIM is compared to a multi-mapping reads resolution tool, MMR. We compare the genome reconstructed by EIM to the genome obtained based on the results of MMR on the read set from $E$. coli $K 12$ and $E$. coli $O 145: H 28$ as a reference. In this way, firstly,

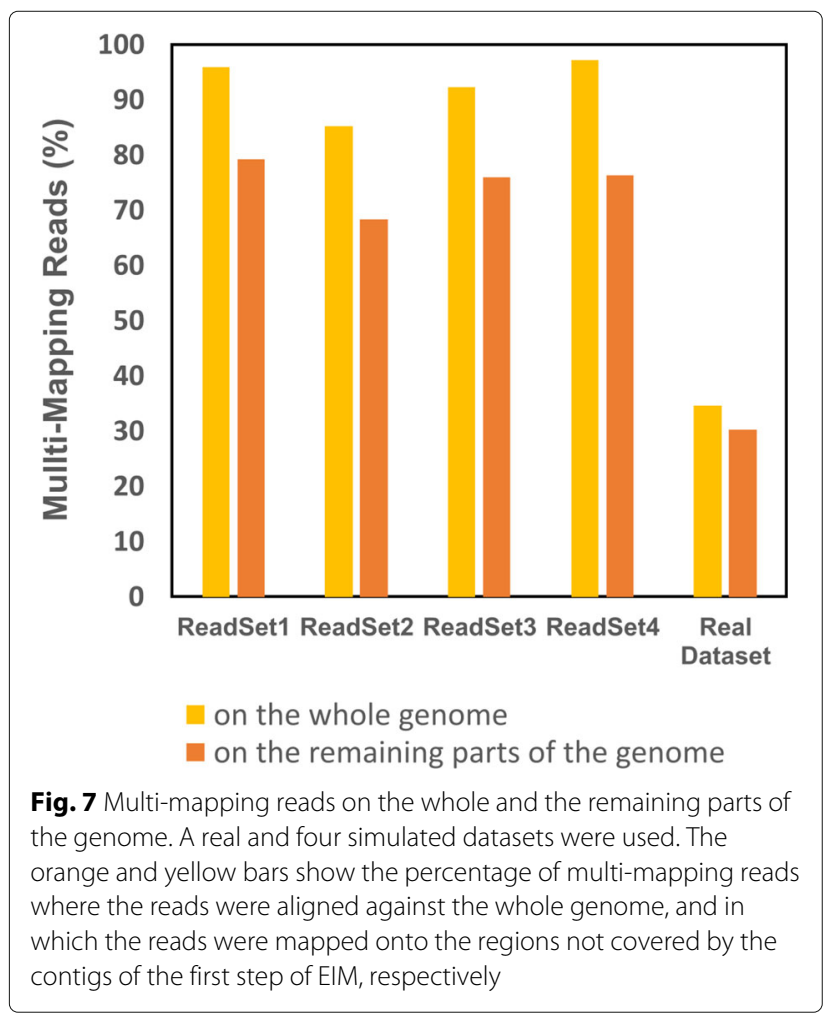

the reads are mapped by Bowtie 2 onto the reference and a SAM file is generated. Then a sorted BAM file and a consensus sequence are built from SAM file as the inputs of MMR and EIM, respectively. MMR produces a BAM file that assigns an optimal mapping location to each multimapping read, while EIM generates a contig set such that the number of multi-mapping reads are decreased.

As shown in Table 9, both approaches maintain the contiguity and reduce the error rate of the input. In addition, EIM can impressively decrease the number of IUPACcodes from 280 to 56 . The running time of reconstructing E. coli genome by EIM ( $330 \mathrm{sec})$ is significantly less than that of MMR (999 sec) without considering the running time of making the inputs. Note that for reconstructing a genome based on MMR results, a consensus construction stage is required after applying MMR which causes to increase the runtime.

As shown by this analysis, the results of EIM pipeline are comparable to a multi-mapping reads resolution tool in terms of the main goal, that is, reducing the error rate of the genome reconstructed by a mapper.

\section{Conclusion}

The goal of our work is to improve the accuracy of contigs generated using NGS read mappers by decreasing their error rate. To achieve this purpose, we design EIM pipeline which aligns the exact and inexact reads against the genome sequence at two separate steps to map the inexact reads more precisely. The assessment of our pipeline on simulated and real read sets show that the separation of reads is effective in reducing the number of mismatch and indel errors with regard to the target genome and significantly decreases the number of IUPAC-codes in the input genome. The evaluation of EIM by three mappers namely Bowtie2, BWA and Yara also indicates that our pipeline, as a post-processing step to different mappers, can improve the genome sequences reconstructed by them in an acceptable running time. In addition, EIM pipeline can reconstruct a comparable genome to that of MMR (a multi-mapping reads resolution tool) in terms of error rate.

Table 9 Comparing EIM and MMR results on a real dataset

\begin{tabular}{llll}
\hline Assembly & Bowtie2 & EIM (v2) & MMR \\
\hline Contigs-500 & 259 & 263 & 260 \\
N50 (kbp) & 29.3 & 32 & 31.3 \\
Errors & 2472 & 1190 & 1369 \\
IUPAC-codes & 280 & 56 & 224 \\
Genome-Fraction (\%) & 88.575 & 88.578 & 88.671 \\
\hline
\end{tabular}

The evaluation metrics has been defined in the text. The columns headed 'Bowtie2', 'EIM ( $(2)$ ' and 'MMR' represent the contiguity and quality of contigs obtained based on the results of Bowtie2, EIM ( $v 2$ ) pipeline and MMR tool, respectively 


\section{Abbreviations}

bp: Base-pair; BAM: Binary Alignment/Map; Chr: chromosome; cns-bt: Genome sequence reconstructed by Bowtie2; EIM: mapping Exact and Inexact reads separately and then Merging the constructed contigs; indel: Insertion or deletion; kbp: Kilo base-pair; NCBI: National Center for Biotechnology Information; NGS: Next generation sequencing; ref: Reference; SAM: Sequence Alignment/Map; SNV: Single nucleotide variant; SRA: Short read archive

\section{Acknowledgements}

Not applicable.

\section{Funding}

This research is supported in part by a grant (No. BS-1397-01-02) from the Institute for Research in Fundamental Sciences (IPM), Tehran, Iran.

\section{Availability of data and materials}

The Illumina MiSeq pair-end read set from E. coli is avialable from [17, 18]. Escherichia coli str. K12 substr. MG1655 and Escherichia coli O145:H28 str. RM12581 are available from GenBank under the accessions NC_000913 and CP007136.1-CP007136.3, respectively. The whole human genome samples are available from the SRA database of NCBI with accession numbers SRR67780, SRR67785, SRR67787, SRR67789, SRR67791, SRR67792, SRR67793. The human reference genome GRCh38 is available from[19]. TAIR10 is available from GenBank under accessions CP002684.1-CP002688.1. Bur-0 strain of Arabidopsis variations respective to TAIR10 reference are available from [22]. EIM pipeline and simulated datasets generated and analysed during the current study are available at http://bioinformatics.aut.ac.ir/EIM/.

\section{Authors' contributions}

FS, FZM, and MS contributed ideas and participated in writing this article. HRZ contributed to choosing datasets. All authors read and approved the final manuscript

\section{Ethics approval and consent to participate}

Not applicable.

\section{Consent for publication \\ Not applicable.}

\section{Competing interests}

The authors declare that they have no competing interests.

\section{Publisher's Note}

Springer Nature remains neutral with regard to jurisdictional claims in published maps and institutional affiliations.

\section{Author details}

${ }^{1}$ Mathematics and Computer Science Department, Amirkabir University of Technology (Tehran polytechnic), Tehran, Iran. ${ }^{2}$ School of Biological Science, Institute for Research in Fundamental Sciences (IPM) P.O. Box: 19395-5746, Tehran, Iran. ${ }^{3}$ National Institute of Genetic Engineering and Biotechnology, Tehran, Iran. ${ }^{4}$ Department of Biotechnology and Molecular Medicine, Zanjan University of Medical Sciences, Zanjan, Iran.

Received: 14 March 2018 Accepted: 11 October 2018

Published online: 06 November 2018

\section{References}

1. Kent WJ. BLAT-the BLAST-like alignment tool. Genome Res. 2002;12: 656-64.

2. Li H, Ruan J, Durbin R. Mapping short DNA sequencing reads and calling variants using mapping quality scores. Genome Res. 2008;18(11):1851-8.

3. Lin H, Zhang Z, Zhang MQ, Ma B, Liy M. ZOOM! zillions of oligos mapped. Bioinformatics. 2008;24(21):2431-7.

4. Homer N, Merriman B, S.F. N. BFAST: An Alignment Tool for Large Scale Genome Resequencing. PLoS ONE. 2009;14(11):e7767.

5. Li R, Li Y, Kristiansen K, Wang J. SOAP: short oligonucleotide alignment program. Bioinformatics. 2008;24(5):713-4.

6. Langmead B, Trapnell C, Pop M, Salzberg S. Ultrafast and memory-efficient alignment of short DNA sequences to the human genome. Genome Biol. 2009;10(3):25.
7. Langmead B, Salzberg S. Fast gapped-read alignment with Bowtie 2. Nat Methods. 2012;9(4):357-9.

8. Li H, Durbin R. Fast and accurate short read alignment with burrows-wheeler transform. Bioinformatics. 2009;25(14):1754-60.

9. Li H, Durbin R. Fast and accurate long-read alignment with burrows-wheeler transform. Bioinformatics. 2010;26(5):589-95.

10. Siragusa E, Weese D, Reinert K. Fast and accurate read mapping with approximate seeds and multiple backtracking. Nucleic Acids Res. 2013;41(7):78

11. Li R, Yu C, Li Y, Lam T-W, Yiu S-M, Kristiansen K, Wang J. SOAP2: an improved ultrafast tool for short read alignment. Bioinformatics. 2009;25(15):1966-7.

12. Gontarz P, Berger J, Wong C. SRmapper: a fast and sensitive genome-hashing alignment tool. Bioinformatics. 2013;29(3):316-21.

13. Lee W, Stromberg M, Ward A, Stewart C, Garrison E, Marth G. MOSAIK: a hash-based algorithm for accurate next-generation sequencing read mapping. PLoS ONE. 2014;9(3):e90581.

14. Kahles A, Behr J, Rätsch G. MMR: a tool for read multi-mapper resolution. Bioinformatics. 2016:32(5):770-2.

15. Li H, Homer N. A survey of sequence alignment algorithms for next-generation sequencing. Brief Bioinform. 2010;11(5):473-83.

16. Li H, Handsaker B, Wysoker A, Fennell T, Ruan J, Homer N, Marth G, Abecasis G, Durbin R. The Sequence Alignment/Map Format and SAMtools. Bioinformatics. 2009;25(16):2078-9.

17. Koren S, Harhay GP, Smith TP, Bono JL, Harhay DM, Mcvey SD, Bergmen NH, Phillippy AM. Reducing assembly complexity of microbial genomes with single-molecule sequencing. Genome Biol. 2013;14(9):101

18. PacBio Corrected Reads (PBCR) Pipeline. http://www.cbcb.umd.edu/ software/PBCR/closure/index.html. Accessed 23 Oct 2018.

19. UCSC Genome Browser. http://hgdownload.soe.ucsc.edu/goldenPath/ hg38/chromosomes/. Accessed 23 Oct 2018

20. DWGSIM. https://github.com/nh13/DWGSIM. Accessed 23 Oct 2018.

21. Huang W, Li L, Myers JR, Marth GT. ART: a next-generation sequencing read simulator. Bioinformatics. 2012;28(4):593-4.

22. 19 Genomes of Arabidopsis Thaliana. http://mtweb.cs.ucl.ac.uk/mus/ www/19genomes/variants.SDI/bur_0.v7c.sdi. Accessed 23 Oct 2018.

23. Gurevich A, Saveliev V, Vyahhi N, Tesler G. Quast: quality assessment too for genome assemblies. Bioinformatics. 2013;29(8):1072-5.

24. Miller JR, Koren S, Sutton G. Assembly algorithms for next-generation sequencing data. Genomics. 2010;95(6):315-27.

25. Laehnemann D, Borkhardt A, McHardy AC. Denoising DNA deep sequencing data-high-throughput sequencing errors and their correction. Brief Bioinform. 2015:17(1):154-79.

26. Zimin A, Marçais G, Puiu D, Roberts M, Salzberg S, Yorke J. The MaSuRCA genome assembler. Bioinformatics. 2013;29(21):2669-77.

27. Magoc T, Pabinger S, Canzar S, Liu X, Su Q, Puiu D, Tallon $\sqcup$, Salzberg SL. GAGE-B: An Evaluation of Genome Assemblers for Bacterial Organisms. Bioinformatics. 2013;29(14):1718-25

28. Walker BJ, Abeel T, Shea T, Priest M, Abouelliel A, Sakthikumar S, Cuomo CA, Zeng Q, Wortman J, Young SK, Earl AM. Pilon: An Integrated Tool for Comprehensive Microbial Variant Detection and Genome Assembly Improvement. PLoS ONE. 2014;9(11):e112963.

29. Zerbino DR, Birney E. Velvet: Algorithms for de Novo Short Read Assembly Using de Bruijn Graphsr. Genome Res. 2008;18(5):821-9. 